\title{
ISPAS: An Intelligent, Smart Parking Allotment System for Travelling Vehicles in Urban Areas
}

\author{
Amit Kumar Tyagi ${ }^{1}$ and Sreenath Niladhuri ${ }^{2}$ \\ ${ }^{1}$ Research Scholar, ${ }^{2}$ Professor \\ Department of Computer Science and Engineering \\ Pondicherry Engineering College, Puducherry-605014, India \\ amitkrtyagi025@gmail.com,nsreenath@pec.edu
}

\begin{abstract}
Parking is limited in almost every metro city (or urban areas) in the world, which leads to several problems like traffic congestion, air pollution, and driver frustration etc. Several enhancements have been made to overcome such problems (or solve such problems). Best one solution is providing parking to vehicles over the road network to reduce total number of vehicles, accidents, and save environment from greenhouses gases etc. In large parking lots, a driver (or vehicle user) may exit (return) from a parking lot without knowing about new vacant spots (that have been vacated by other users). Besides that, finding an empty parking spot may lead to driver frustration. If some space (a parking lot) is available in parking lot (response by service provider) and this information is passed to vehicle users (by service provider or group vehicles) who are waiting for a parking lot (reside at different location or same distance). If another vehicle occupy that spot before the actual driver, first user will think, service provider response was not right (about this slot). So trust issue raised here. Also due to system updating, parking system require large time to update vacant parking slots, which is also a serious concern. Several approaches (or schemes) have been proposed in past to solve parking problems for urban area but none of them is efficient to provide reliable and efficient service to vehicle user. The contribution of the work is to includel provide the real time parking navigation service and friendly parking information service to the drivers. So to solve such problems, we use a novel approach. In our work, Vehicular Ad Hoc Network (VANET) provides an intelligent service-oriented parking allotment which improves parking space utilization and reduces drivers' frustration over the road network. This work fills this gap, i.e., providing parking demand using vehicular network in consideration. Using our Intelligent and Smart Parking Allotment System (ISPAS), drivers can easily and quickly get their preferred parking lots close to their destination. The proposed system will address the performance analysis via simulation demonstration, efficiency and results. Therefore our results show that, the parking navigation is convenient and efficient, and increases the space utilization in the parking area.
\end{abstract}

Keywords: Parking System; Information Dissemination; On-Board-Unit; Road-Side Unit; Trusted Authority; Vehicle User and Urban Areas

\section{Introduction}

Today world population is above 7 billion and every $20^{\text {th }}$ human being (approximately) consist his vehicle. Each country has its own transport ministry, under which millions of vehicles are running over the road. So at the end of traveling, every user needs a space to park his vehicle near to his destination (or away but should be convenient). Finding a vacant parking space in a congested area or a large parking lot, especially in the peak hours is

Received (September 2, 2017), Review Result (November 21, 2017), Accepted (December 1, 2017) 
always time consuming and frustrating to drivers. Everyday several vehicles (or user/ driver) waste their time and fuel in (circling for) fining a vacant a parking lot. In the past decade, to minimize hassle and inconvenience to the drivers, many parking guidance systems have been developed. Previous proposed works has tried to provide accurate, realtime car park space availability to the drivers. Users who are looking for parking spaces, Previous works guides to users to provide a reliable and convenient palace. The current parking guidance systems (as in existed works) obtain the availability of parking spaces using the sensors installed across the whole parking lot. However, as a disadvantage of existed work, deploying sensors in a large parking lot are very expensive. Furthermore, the sensors can become inaccurate and would stop functioning easily when time passes. Therefore, it is highly desired to have a reliable and cost effective way to track available parking spaces and guide drivers to the available parking spaces. Besides searching for available parking spaces, if the driver have the up-to-date knowledge about the traffic situation like information about the free (vacant) parking place near the destination area, it would be then a great benefit (market segmentation) for him.

Unlike Mobile Ad hoc Network (MANET), vehicles move on predefined roads, vehicles velocity depends on the speed signs and in addition, these vehicles also have to follow traffic signs and traffic signals. Several challenges needed to be solved (in VANET) in order to provide reliable services to vehicle over the road network. Stable \& Reliable routing (used for passing information to users) in VANET is one of the major issues. Security and privacy of drivers and vehicles is also a major issue in VANET but this work mainly focus only on stable and reliable routing. As vehicles have dynamic behavior, high speed and mobility that make routing even more challenging. VANET present a promising way to build up a decentralized parking guidance system. Designing such an application can be decomposed into major issues: (a) Which information on a parking place needs to be known by the vehicles and thus has to be distributed in the VANET (b) How can this information be used to maximize the benefit for the driver?

Vehicle Ad Hoc Networks (VANETs) has been received attention both in industrial and academic levels [1]. With the advance and wide deployment of wireless communication technologies, many major car (vehicle) manufactories and telecommunication industries gear up to equip each vehicle with the On Board Unit (OBU) communication device, which allows different cars (vehicles) to communicate with each other as well as roadside infrastructure, i.e., Road Side Units (RSUs), in order to improve not only road safety, but also better driving experience. Therefore, it becomes possible to track the parking space occupancy, guide drivers to the empty parking spaces in large parking lots through vehicular communications. When a user request for a vacate parking space from application provider, then user need to give his basic information (like name, age, vehicle number etc.,) to service provider (at the time of registration or issuing ticket). Then service provider may reveal this information to unauthorized users or other organisations i.e., issue of privacy of a user raised here. To solve such issues or problems, we need an intelligent and smart parking system.

\subsection{Advantages of Parking based Information}

Principally reusing of parking spaces (retroflex or replication) is a proactive job. This means that before vehicle needs and only based on future access probability, the data are transferred to vehicles or its access environment, or else, there would not be any difference between replication and data dissemination [10]. Thus, vehicle's (in parking) parking time is a suitable opportunity to replicate data to reveal his identity in future. The present methods have categorized replication in moving vehicle's group and the prediction of isolation of vehicles from the group. Exchanging Data (replication) in parking lots has some features that increase replication advantages. Several benefits have been mitigated in parking scenario, like: 
- Vehicles' moving in groups is short particularly in urban territories and there is insufficient time to make decision about fitting information for replication and trade. Long term stop of vehicles in stopping pre-pares enough time to run ideal calculations to choose fitting vehicles for information replication.

- It is uncommon for moving vehicles to meet later on once more. Subsequently, imitated information would not be valuable after the vehicles gathering is broken down. Be that as it may, visit stops of a vehicle in a stopping would expand the likelihood of reusing the recreated information.

- Frequent change of individuals in moving groups will request a few replications and it authorizes heaps of expenses to the system. In the mean-time, replication in a couple of vehicles in a stopping would be sufficient to make certain the information is always accessible.

- The available transmission capacity for VANET is exceptionally restricted particularly in high movement zones. The move of non-emergent forms like replication to vehicle's stop time in stopping will lessen activity heaps of VANET and would help safety and driver assistant applications.

- Owner of vehicles that are parked in an area or location usually share same components and their information are legitimately related. For instance, they have common residence of workplace or group. Along these lines, the likelihood (probability) that they are comparative in regards to information needs is high, either. Therefore, searching data or information in vehicles stopped in neighborhood would prompt to better results.

- When vehicles are parked they would have more opportunity to gather and oversee comparable demands and distribute normal information between vehicles utilizing communicate packets that result in a reduction of network traffic.

So in summary, advantage of providing efficient parking slots to vehicle users helps in reducing/ avoiding network congestion (over the road network), saving fuels or gases to protect environment. To achieve various mitigated goal, following four issues need to be addressed:

- How to manage the parked vehicles within a parking lot to facilitate parking guidance?

- How to obtain the internal map of a parking lot?

- How to achieve effective parking navigation inside the parking lot?

- How to realize efficient parking availability information dissemination outside the parking lot?

The rest of this paper is organized as follows: introduction section discusses need of parking, advantage and various goals to achieve in VANET applications. Section 2 discusses about related work done in respective (applications) schemes. Section 3 describes our problem in brief. Section 4 draws design goals and models with proposed algorithm. In Section 5, this work mentions some performance metrics and experimental setup with efficient results. Finally, Section 6 concludes this work with a summary of contributions. In the following sections, we do not differentiate terms "vehicle," "car", "object," "users "and "moving object".

\section{Related Work}

With considering safety (reliable) and stable services as essential one, now day's many vehicle manufacturing companies construct (or provide) vehicle with the enabled On Board Unit (OBU) communication devices. To solve parking problems, several attempts have been made by several researchers in the past decade. In Wireless ad hoc discovery of Parking Spaces they have proposed a scenario of wireless ad hoc networks for finding vacant parking places. They use multi-hop dissemination of information only among interlinked parking meters (i.e., but not among vehicles). Requests from vehicles for vacant parking places are received and handled by such a parking meter via single-hop 
communication In Smart Parking, an Application of optical Wireless Sensor Network (WSN), authors present a Smart Parking Management System (SPMS) based on wireless sensor network technology, which provides remote parking monitoring, automated guidance and parking reservation service. In [4], Gongjun et al. proposed a wireless-based intelligent parking system especially applied to large parking lots. This parking system uses infrared sensors to sense vehicle movemnets and parking belts to control vehicle entry and exit. A parking system and the OBU use the surround sensors (mounted on vehicle bumpers) to enhance the system's context-aware characteristic and then uses the collected information to calculate the size of the available space in a parking area. Verroios et al. [5] proposed a parking reservation system by deploying sensors on vehicles. Panyappan [3] proposed a parking system that reduces the extensive and costly deployment infrastructure, and the available parking lots can still be located effectively. This proposal was restricted to parking lots. Kenny [6] emphasised various applications that widely use DSRC also consider OBU and IS (info-station). Info-station is regional servers (established in each parking areas) or some intermediates to provide correct information about vacant parking slots. Info-stations or observe entering or leaving time of a vehicle user in parking area. These location based (or VANET) applications primarily consist of collision prevention and collision avoidance functionality.

Ghazy and Ozkul [7] integrated VANET with a RSU to use DSRC to solve traffic congestion problems, i.e., to provide safety and a comfort services to drivers. We need to reduce congestion/jamming problem over the road network (for people's safety) with developing some application for vehicular network. This application alleviates many unfavourable effects with respect to the health of the community, the economy and the environment during longer commutes. But work done in [7] took care of the traffic congestion issue. Jie Sun et al. [8] proposed a sensor-based context-aware smart car that imparts a 'complex reasoning' faculty. The approach discussed in [11] disseminates aggregated parking information about areas that cover a lot of parking terminals, while taking the local relevance and age of information into account. In [12] the proposed protocol does not disseminate the same information to all vehicles. Vehicle self-coordination is also discussed in [14] where a protocol for traffic flow management in intersections is suggested. In [15], authors discussed a decentralized and collaborative approach to bypass congested areas in large road networks based on information disseminated among moving vehicles.

In [13], authors predict the occupancy of a parking lot, by modeling it as a queue and using a Markov chain. Another work related to problem of finding a vacant parking space is discussed in [2], where vehicle-to-vehicle $(V 2 V)$ and vehicle-to-infrastructure (V2I) communications are used to help vehicles navigate inside a large parking lot. Further two basic approaches for the parking space search problem are also investigated in [16]: (a) a decentralized approach that uses $V 2 V$ communication in order to disseminate parking space information among vehicles and (b) a centralized approach where a server acts as a reservation system for vehicles' parking requests. The system presented in [17], consists of a central server that collects reports for parking spaces detected by vehicles' sensors, in order to produce a real-time map of parking space information. Parking space allocation and routing to a reserved space is provided by the system proposed in [18], using aggregate parking and traffic information from vehicles and roadside units. Further, Lu et al. [19] propose a smart parking scheme, called SPARK, to provide convenient parking services in large parking lots. The scheme employs three Road Side Units (RSUs) deployed in the parking lot to monitor and manage the whole parking lot using the VANET communication technology. The main advantages of SPARK include providing real-time parking navigation service to drivers in large parking lots and friendly parking information dissemination service to the moving vehicles. Considering the large amount of investment and elaborate design of RSU-based approaches, Zhao et al. [20] propose an infrastructureless parking guidance scheme, called IPARK, for urban areas [11]. The IPARK provides drivers two main services, including parking availability information dissemination outside 
the parking lot and real-time parking navigation in the parking lot. Without any infrastructure investment, the IPARK is actually a cost-effective scheme. The performance evaluation results also show that IPARK can achieve effective parking guidance. Hence many approaches have been explained to provide an intelligent a smart parking over the road network, but no approach concern user's privacy in their proposal.

Hence this section discusses about various attempts made by several researchers all around the world. Now next section will dealt with various parking design goals and models in details.

\section{Problem Description}

Revealing the POIs of a particular user is likely to cause a privacy breach as this information may be used to infer sensitive information such as hobbies, religious beliefs, political preferences or even potential diseases. Losing of stored information with service provider (collected by service provider during providing services), if shared with other vehicle user without user's or owner permission, then trust is not more existed between third party and vehicles users. Further when a computational infrastructure informs the vehicle about available parking, but the vehicle need to determine the exact time to reach at the parking space (destination) because that parking space may occupied by another vehicle (in this meantime) and no provision exists to inform the first vehicle about this situation. Here validity of information (exchanging with user and service provider) is a major issue. By the way, user is only responsible person for revealing/leaking his location privacy attributes. Many user uses several applications in location based services without reading privacy policy agreement (provided by application provider). Hence a user is only responsible for losing his information while accessing location based series over the road network [23, 24].

To solve above problems, we proposed a payment-based architecture in which, once a vehicle books a parking space, other vehicles cannot use that parking space. We extend work of [3] by a further reduction in infrastructure and apply the architecture to on-street parking zones that demand a slightly more complex management structure compared with parking lots. Also we extend the concept to ISC, which was presented in [9] and utilise the high capacity DSRC channel for the comfort application of an on-street/ road parking reservation. For that an Intelligent GPS-based Vehicle navigation system for finding vacant parking slots over the road/street networks has discussed in algorithm 2 and 3 (in section 4). Our results show that, we provide the most likely available parking lots to users within a specified distance (from a user's final destination). The proposed algorithm utilizes the occupancy history and the present status of a parking lot in order to determine its availability at the time when the vehicle would likely reach the destination (arrival time) and departure time from destination (parking slots). Our proposed work expresses the quality of a trip that visits reported parking places in a sequence using a cost function. Our VANET-based architecture (i.e., ISPAS) focused only on the parking spot location and reservation (of same size or type like car) without considering congestion issues. Our approach provides accurate (updated) response to users regarding a vacant parking slot in minimum time. Note that in our proposed architecture, the need for sensors on the parking spaces is eliminated (due to preservation of user's privacy). For removing or probability of being tracked with GPS, we do not use sensors devices in our work. With this action, we preserve the actual location of a user (vehicle) over the road network.

\section{Parking Design Goals and Models}


In real world, Privacy is a major issue than confidentiality. It is about the provision for any person, or any piece of data, to keep (hide) information about themselves from others (outside world). Trust is a key attribute of security architecture. Trust is an essential element [21] to preserve privacy of vehicle in parking [21]. As discussed in introduction section, to provide the convenient parking services to the driver in the parking lots, we assumed the TA (Trusted Authority) which will be the registration in-charge of both RSU and OBU to keep the secrets [22] of RSU and OBU (if any exceptional event occurs). Vehicles are equipped with the sensing computing and wireless devices.

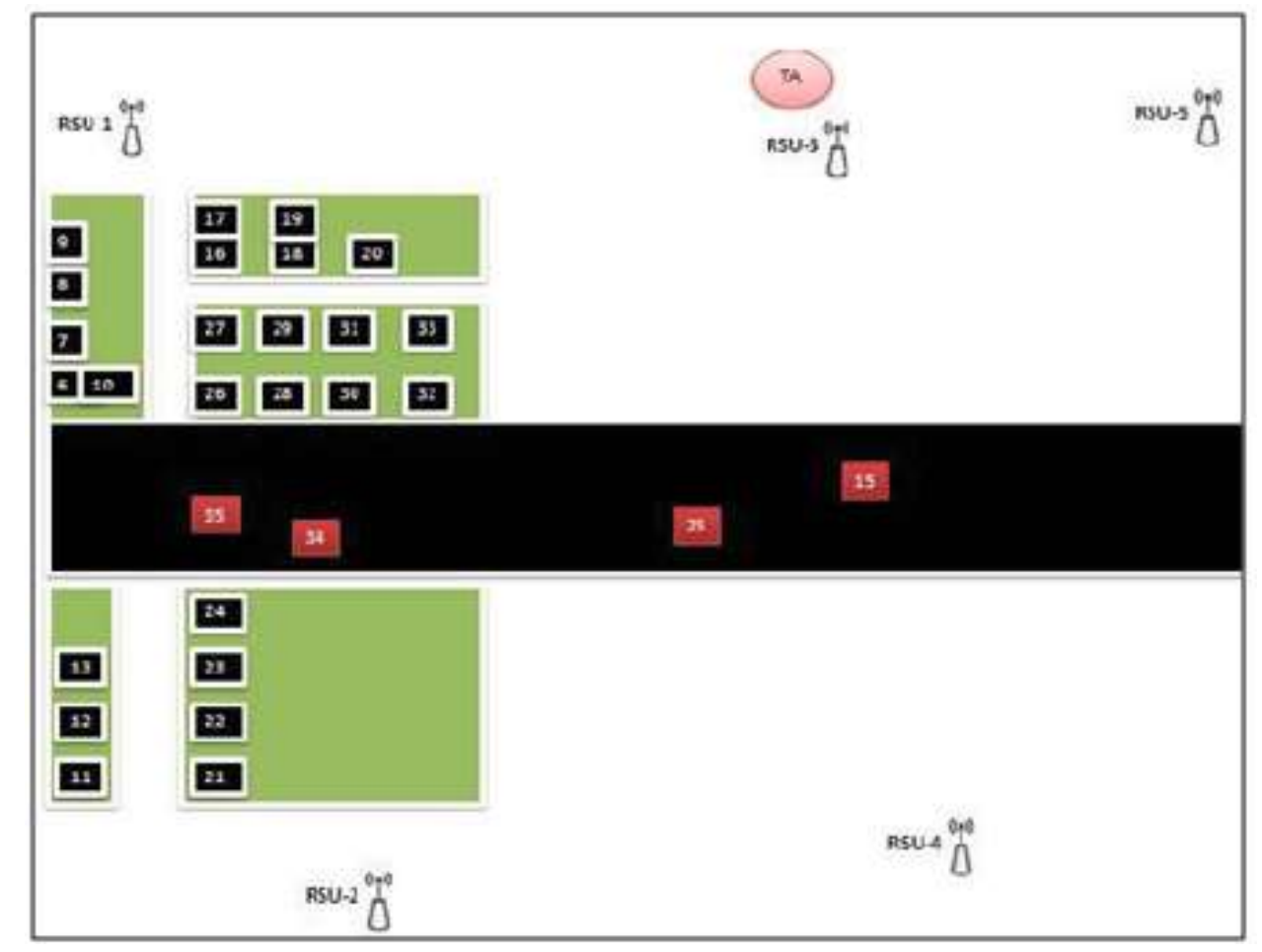

Figure 1. A Typical Parking Lot Model under Consideration for RSUs

Note that this work does not provide any privacy preserved concept for vehicle user's/ or his information which is stored in parking system database or trust enhancement concept among users, it just provide an efficient parking space with minimum walking distance and cost to users in urban areas with enough security, i.e., using security tools like CCTV cameras. Also this work provides parking to same size of vehicles only. The RSUs are installed in the parking lot to get the request message from OBU and to provide the correct information about the vacant parking space back to the OBU. The Parking system makes the following contributions:

- The system can provide real-time parking navigation service to drivers in large parking lots. With the real-time parking navigation, the drivers can get the vacant parking space quickly. Therefore, the gasoline and time wasted in search of vacant parking space can be reduced. To the best of our knowledge, this is the first such effort in the context of VANET-based real-time parking navigation.

- The system can provide friendly parking information dissemination service to the moving vehicles. With this friendly parking information, the drivers can conveniently and quickly choose their preferred parking lots close to their destinations (market segmentation).

In general, in parking guidance system, the vehicle users can also communicate with other vehicles within its range to provide a sufficient and reliable parking without the help 
of RSU or any authority. But in case of availability of any free space (which is informed by other neighbour users), other users who are wishing for a parking space, need to register with the respective authorities to reserved that particular space. In other case, Vehicles in some destination areas have several choices amongst several possible parking opportunities. Figure 2 shows that a vehicle has different choices to park his/ her vehicle (communicating under three different RSUs). We assure that, our phenomenon in provide a friendly parking system to vehicle users. Figure 1 and 2 shows a clear picture about typical parking lot with considering RSUs over the road/ street network.

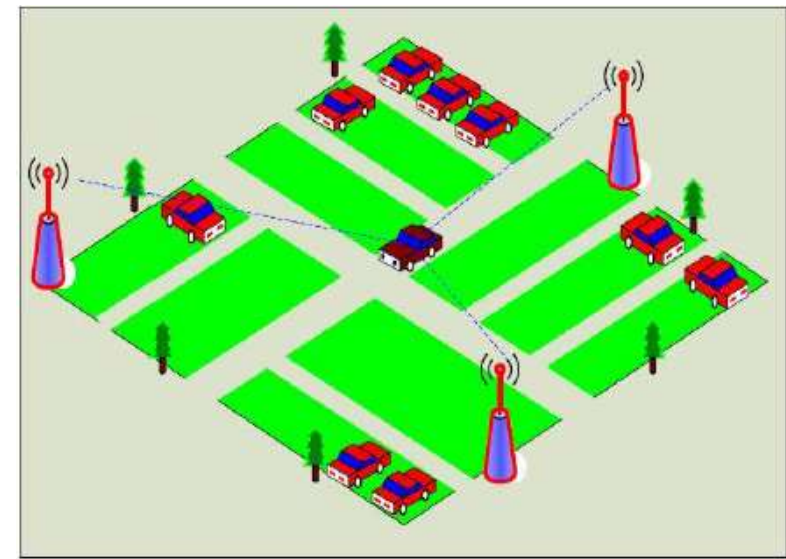

\section{Figure 2. A Typical Efficient Parking Lot under Consideration for RSUs}

In Figure 1, we see five RSUs connect and communicate with each other to provide a reliable, efficient and friendly parking services to vehicle user over the road/ streets/ specific places. In parking, vacant or non-vacant space for a vehicle can be computed as:

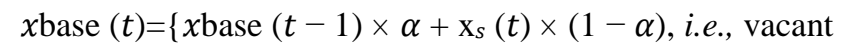

where $\alpha$ is the forgetting factor which denotes the update frequency of baseline. And the $(t)$ is the filtered value of sensed signal. A system is updated only when the parking space is vacant. The $y$-axis and $z$-axis can be processed in a similar way.

Now, if user $i$ arrives at time $t$, then $\mathrm{K}_{\mathrm{t}} \varepsilon\{0 \ldots . . \mathrm{K}\}$ parking spaces are unoccupied at $\mathrm{t}$ and $\mathrm{n}_{\mathrm{t}} \varepsilon\{0 \ldots \mathrm{N}\}$ users (drivers) arrive at $\mathrm{t}$, his payoff is:

$$
\mathrm{U}_{\mathrm{i}, \mathrm{t}}=\mathrm{P}_{\mathrm{i}, \mathrm{t}} \mathrm{V}-\mathrm{L}(\mathrm{t})
$$

A user who arrives at time $\mathrm{T}$ and finds no parking space must park in a less convenient lot, obtaining pay-off zero. A user who arrives at $t^{*}$ and finds a parking space receives a payoff of zero since the disutility of the early arrival equals the value of the parking space. When looking for equilibria, we can restrict our attention to $t \geq t^{*}$ since arriving before $\mathrm{t}^{*}$ yields a negative payoff and is strictly dominated by arriving at $\mathrm{T}$.

In our (an essential feature) of ISPAS System, some parking spaces (i.e., 20\%) are reserved for emergency or handicapped or old people etc. purposes. Further as another essential feature, this work provides observation of vehicles at the time of parking of vehicle at IN and OUT time (called Reference Irregularity) (see Figure 3). To calculate this parameter (Reference Irregularity) initially the time range of the presence of a vehicle in the parking is gained from the average entering times (IN) and exit time (OUT) (refer equations 2-5) to the parking. Hence, in equations $2-5$, we can calculated vehicle's in (arrival time) and out (departure time) timing to protect from some incidents. Basically, 
this feature used to prevent (or assuring users attendance or presence in parking system) from any malicious or suspicious activities/ incidents. For example, if users buy ticket of parking space to park his vehicle but he did not park his vehicle at that time/ day. And he moved to somewhere else to perform such illegal things like any murder via hit and run. So in any police case, he can come clean because he can tell to authorities that my vehicle was not available at that location because it was parked at particular location. But actually it was not. That user is lying to authorities. To prevent such incidents, we provide observing of vehicles at IN and OUT time. We provide sign-in and sign-out feature to each user whoever is interested for or need parking slots over the street/ road network.

$$
\begin{aligned}
& \text { Duration }=\frac{\sum_{k=1}^{\text {day count }}(\text { day staying })}{\text { daycount }} \ldots \ldots \ldots \ldots \ldots \ldots \\
& {[\mathrm{IN}(\text { Irregularity })]^{2}=\frac{\sum_{k=1}^{\text {day count }}(I N k-I N)^{\wedge} 2}{\text { daycount }} \ldots \ldots \ldots} \\
& {[\text { OUT }(\text { Irregularity })]^{2}=\frac{\sum_{k=1}^{\text {day count }}(\text { oUT }- \text { OUT })^{\wedge} 2}{\text { daycount }}}
\end{aligned}
$$

Ir reguralty $=[\mathrm{IN}($ Irregularity $)+$ OUT(Irregularity $)] / 2$

Since these equations would finally lead to recognize resident vehicles with long stop duration in the parking, to eliminate the effect of single irregularities occurred regarding the presence of a vehicle in the parking, those references that have had a strong standard deviation from in-out times form predicted ones are deleted. The irregularity of any vehicle is calculated as the average of standard deviation of vehicle's daily entry and exit times in a parking. Cooperation of moving vehicles and those parked in the road side would supply a consistent structure for communication between moving vehicles in the absence of RSUs.

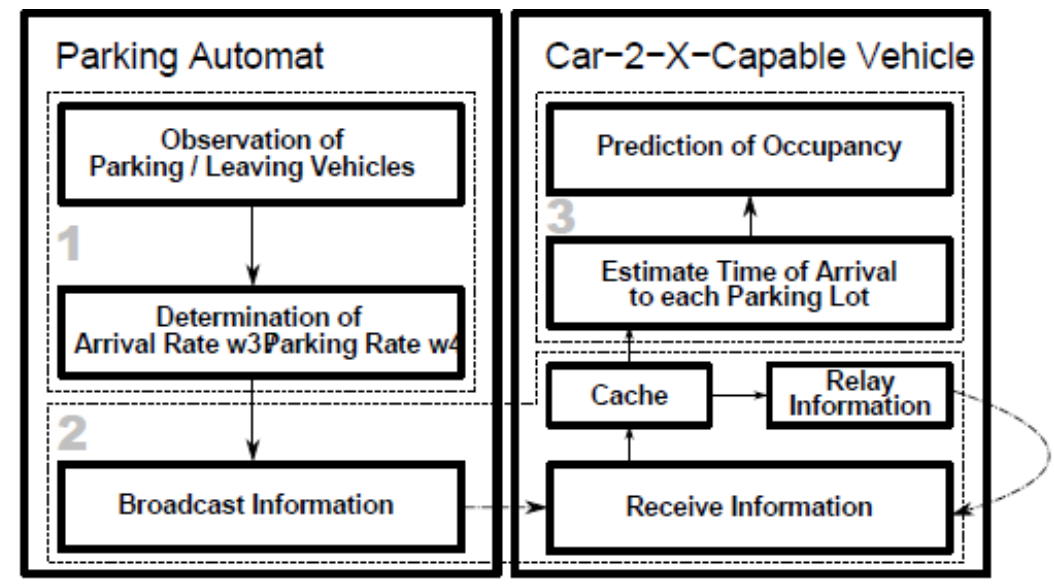

Figure 3. Information Flow in Predicting Parking Lot Occupancy

Now proposed system related to Figure 3 can be discussed in algorithm 1-4.

Algorithm 1: This algorithm is used to select users for a parking space (with multiple parking choices for users) (see Figure-4)

1. Set up a large parking lot with identifier IDj,

2. Each parking space is designated a location $\left(\mathrm{x}_{\mathrm{i}}, \mathrm{y}_{\mathrm{i}}\right)$,

3. Three parking lot RSUs of the same height $\mathrm{h}$ are erected at the locations $(0,0),\left(\mathrm{x}_{\mathrm{a}}, \mathrm{y}_{\mathrm{a}}\right)$ and $\left(\mathrm{x}_{\mathrm{b}}, \mathrm{y}_{\mathrm{b}}\right.$ ), respectively. (Whole parking lot will be under surveillance of these three RSUs), as shown in considered Figure. 
4. After TA inspects the parking lot, $\mathrm{TA}$ generates the private key $\mathrm{skj}=\mathrm{sH}(\mathrm{IDj})$

5. Stores the same private keys skj into the three RSUs.

6. A large smart parking lot is established.

7. End

Algorithm 2: Selection of user with respect to parking space.

1. Identify location of vehicles (same types) over the road networks/ area

2. Make clustering of vehicles according to your requirement but keep at least 5 vehicles in a cluster, while maximum users keep 25.

3. Identify total parking spaces available near to created clustered vehicles

4. Provide detail about vacant/ free parking spaces to respective vehicles users

5. Either any time, any user can ask/ contact their surrounding neighbours about free parking slots in their area

6. Get response from users notify to central authority

7. Receive confirmation/ parking ticket form authority

8. Provide/ deliver ticket to concern user with an expiration time

9. Provide final walking space from parking space to user's destination (apply algorithm 3)

10. Provide live mode parking (put registering entering time in parking time) (apply algorithm 4)

11. End

Basically, this algorithm used for provide a spot in a parking slot, i.e., in a case when a driver wants a parking slot (from his colleagues or neighbours). In this case, user needs to go in person to the parking lot and after paying some parking fee and basic information about himself or vehicle (to private parking owner/ authority/service provider). He can collect parking tickets from respective authority to park his vehicle in respective parking lot (for particular information). Note that collected information can be stolen or revealed by service provider. But after a period, when this information expires, there is no meaning of this information.

Algorithm 3: Optimal Trip Based on $\mathrm{C}(\mathrm{a}, \mathrm{b}, \mathrm{t})$. This algorithm provides free parking slots based on minimum walking distance and cost.

Input: ParkingSpaces (PS): The set of parking spaces inside the maximum walking range, $\mathrm{T}$ : The time of the longest trip that visits all the parking spaces

Output: The trip that starts from the current position, visits all the spaces, and has the minimum cost

Begin

1. $\mathrm{s}:=$ vehicle's current position/ location

2. for each space $y \in$ ParkingSpaces do

3. time $:=$ time to drive from $\mathrm{s}$ to $\mathrm{y}$

4. $\mathrm{B}(\mathrm{s}, \mathrm{y}, \emptyset$, time $):=\mathrm{C}(\mathrm{s}, \mathrm{y}$, time $)$

5. store $\mathrm{s}$ as the predecessor of space $\mathrm{y}$ in $\mathrm{B}(\mathrm{s}, \mathrm{y}, \emptyset$, time $)$

6. end for

7. spaces $:=\mid$ ParkingSpaces $\mid$

8. for size $=2,3, \ldots$, spaces do

9. for each subset of spaces $\mathrm{S}$ such that $|\mathrm{S}|=$ size do

10. for $\mathrm{t}=1,2, \ldots, \mathrm{T}$ do

11. for each space $y \in S$ do

12. for each space $x \in S-\{y\}$ do

13. time $:=$ time to drive from $\mathrm{x}$ to $\mathrm{y}$

14. $\mathrm{u}:=\mathrm{t}-$ time

15. if $B(s, x, S-\{x, y\}, u)$ is equal to $\infty$ then 
16. continue with the next space $x$

17. end if

18. addedCost $:=\mathrm{C}(\mathrm{x}, \mathrm{y}, \mathrm{t})$

19. if $\mathrm{B}(\mathrm{s}, \mathrm{x}, \mathrm{S}-\{\mathrm{x}, \mathrm{y}\}, \mathrm{u})+\operatorname{added} C o s t<\mathrm{B}(\mathrm{s}, \mathrm{y}, \mathrm{S}-\{\mathrm{y}\}, \mathrm{t})$

then

20. $B(s, y, S-\{y\}, t):=B(s, x, S-\{x, y\}, u)+$ addedCost

21. store $x$ as the predecessor of $y$ in $B(s, y, S-\{y\}, t)$

22. end if

23. end for

24. end for

25. end for

26. end for

27. end for

28. bestCost $:=\infty$

29. for each space $y \in$ parkingSpaces do

30. $\operatorname{cost}:=\mathrm{B}(\mathrm{s}, \mathrm{y}$, parkingSpaces $-\{\mathrm{y}\})$

31. if cost $<$ bestCost then

32. bestCost $:=$ cost

33. end if

34. end for

35. return the trip that refers to bestCost

End

Note that in an efficient parking, minimum walking distance (from a parking space to his destination) required by a user/ driver. A user needs parking for his vehicles near to his destination area (based on user's comfort). For example, if a user going to visit a doctor then he need a place to park his vehicle near to respective hospital, not far away from that hospital.

\section{Algorithm 4: Live-Mode Parking System}

Initialization: synchSeq := apply Alg. 2 with the known parking spaces as input curSeq $:=$ synchSeq

\section{Begin}

1. while true do

2. if there is an addition or deletion in the set of known free parking spaces then

3. for each change do

4. if a space $\mathrm{s}$ was taken then

5. affectedCluster $:=$ The cluster that contains $\mathrm{s}$

6. curSeq $:=$ curSeq $-\{$ affectedCluster $\}$

7. end if

8. if a new space $s$ is available then

9. affectedCluster := The best cluster to contain $\mathrm{s}$, based on SF

10. if no cluster could contain $\mathrm{s}$ then

11. affectedCluster $:=$ A new cluster that contains only $\mathrm{s}$

12. else

13. $13:$ curSeq $:=$ curSeq $-\{$ affectedCluster $\}$

14. end if

15. end if

16. insert affectedCluster into the position of curSeq that gives the best cost for the corresponding trip

17. update curSeq with the new insertion

18. end for

19. distance $:=$ The distance between the trips for curSeq and synchSeq

20. if distance $>$ SynchThres then 
21. synchSeq := apply Alg. 2 with the known parking spaces as input

22. $\operatorname{curSeq}:=$ synchSeq

23. end if

24. end if

25. end while

End

Therefore, in order to evaluate the effects of the main parking management strategies, it is necessary to estimate other features to affecting parking schemes. The actions undertaken in the parking management in an urban area included in [2]. Basically, Responsibility of the proposed parking system (ISPAS) concept is: Parking availability information passing on an ad-hoc network, Recognition of authenticated Vehicles and Path changing of vehicles on unavailability of parking space. This section provides a clear design/ model including algorithm or steps to provide efficient parking schemes. Next section discusses about metrics used in count to performance, respective results of proposed approach.

\section{Experimental Setup}

The aim of our work is clearly defined and explained in previous sections. To provide live parking slots (using OBSs, RSUs, and TAs) to users (from different- different locations), we need minimum parking cost and walking distance (with sensing their arrival and departure time). For that simulation and Evaluation is performed by simulating vehicles on a grid road network, i.e., of $1.5^{*} 1.5 \mathrm{mile}^{2}$ (in area). Available parking spaces are mapped to intersections, for a total of 40 spaces and Vehicles are placed on the grid at random locations. Broker (mediator or group) vehicles moved continuously to random waypoints, while consumers traversed a randomly selected search path (a square). Our Tests are performed across a range of broadcast sizes (1-40 reports), numbers of vehicle users (1050), parking unavailability times (10- $30 \mathrm{~min})$, parking system densities (50-200 vehicle per sq. mile), and transmission ranges (50-300 meters). The time between broadcasts is fixed at 5 seconds, mean vehicle speed is $20 \mathrm{mph}$, and $50 \%$ of users are participating. Total simulated time is 30 hours for each of the 40 tests. Our results are compared to ranking by age (or distance) alone, and existed the parking availability system. The average parking discovery time is measured for each participating user. This metric measured the average time of a vehicle, whoever is looking for parking. This is compared with the case of no report dissemination (i.e., blind search). Our results show that our proposed method can successfully learn an optimal combination of relevant attributes for estimating relevance (see Figure 4). Let a system model consisting TA, OBU (equipped in the Vehicles) and stationary RSU (deployed in the parking area). Our approach provides an efficient, reliable parking slot like;

- Once the vehicle goes into the stopping zone the recognizable proof ought to kept secret for an exceptional event occurs. So the RSU can get the data about the enrolled vehicle from the TA. TA is the enlistment responsible for the both RSU and OBU. TA first instates the required parameters. TA reviews the parking garage/ lot and creates the private key (skA) and stores similar private keys into the (near-by) 3 RSUs.

- OBUs are communicated with each other (with OBUs) and with the RSUs to get information (status of) a vacant (free) parking slot the present parking information. Each OBU has given the novel ID, i.e., vanet_ID. To secure the protection of the OBU each vanet_ID (IDv). When OBU with IDv registers to TA, TA changes over the IDv into PIDv and produces the private key Sk. At the point when OBU goes into the parking area, it will get the ticket ID and the ticket key which is known to driver (vehicle user) only. 
- For example, we deploy 3 RSUs in the parking area. These all RSUs cover all the parking area over the street/ road network. All these parking area are assessed by the TA. TA will produce private key for identifier and circulate them to all RSUs.

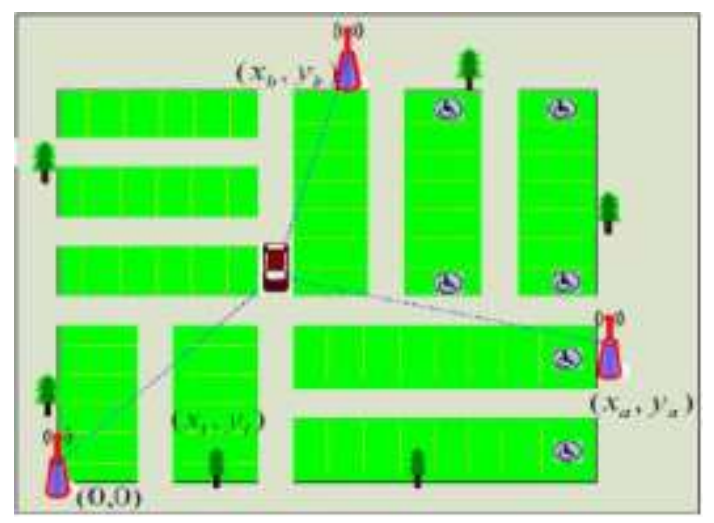

Figure 4. Parking Lot Model under Consideration

Note that above discussed 3 points $\left((x, y),\left(X_{a}, Y_{a}\right)\right.$ and $\left(X_{b}, Y_{b}\right)$ also can be summarized as algorithm 1. Here a communication made up among several entities, which discuss several steps, i.e., Communication between the OBU to OBU and OBU to RSU and vice versa. All steps can be discussed as:

- Ask Parking Request To RSU (from OBU): The driver who wants to park his vehicle send the parking request to RSU, with vanetID, current timestamp, and current position (rbc_posx, rbc_posy). The request (for parking slot) is sent by the OBU to RSU.

- Receiving the parking request from $O B U$ : RSU will receive the parking request message from OBU with the header information $r b c_{-}$senderID, and then RSU will reply back to the $\mathrm{OBU}$ by calling the Send_Parking_Instruction_To_OBU $\left(r b c \_s e n d e r I D\right)$. By searching the available parking point from getting response via the respective authority (or parking provider) and update the routing information of the parking lot with the current time-stamp.

- Identify nearest parking lot: When the OBU enters into parking lot the RSU choose the proper vacant parking points (lot_x, lot_y). These five RSUs synchronically measures the distance from the vehicle to themselves. With the vacant parking points (lot_x, lot_y) and the position of the vehicle (X, Y), the RSU choose the shortest path for the vehicle and navigate the vehicle to the vacant parking space. To calculate the nearest parking points for the requested $\mathrm{OBU}$ following formula (equation 6) is used:

$$
(\text { Nearest distance })^{2}=\left(\mathrm{x}-\mathrm{x}_{1}\right)^{2}+\left(\mathrm{y}-\mathrm{y}_{1}\right)^{2} \ldots
$$

Our goal is provide parking lot near to user's our destination (or with minimum walking time). To achieve this essential requirement, we use equation 6.

- Send Parking Instruction To OBU (nsaddr_t nid): After finding the vacant parking position points at the parking lot with nearest parking points, this parking point information (with the parking lot ID and parking points $(\mathrm{x}, \mathrm{y})$ ) is sent to the respective $\mathrm{OBU}$ (from where the parking request is received).

- Receive the Parking instructions from RSU: Requested (respective) OBU receives the parking information from the RSU about the free parking points $(x, y)$ and Parking_lot_ID_id.

- Send the parking reply to $O B U$ : With the parking instruction the RSU will send the parking reply to the OBU with the RSU_ID and the parking points (x, y).

- Receive the parking reply from $O B U$ : Finally after receiving the parking points by the $\mathrm{OBU}$, the driver will park the vehicle at the respective parking points. 
- $O B U$ registration at TA: In our considered scenario, every OBU will registration itself at TA (by sending the registration message to TA with his vanetID, and current timestamp).

- Receive registration message by TA: After receiving the registration message from OBU, TA will do the registration of the OBU and generate the private key (skA) for the OBU. Once the vehicles enters in a parking area. An exceptional event occurs which identifier should be secret. So the RSU can get this information about the registered vehicle from the TA.

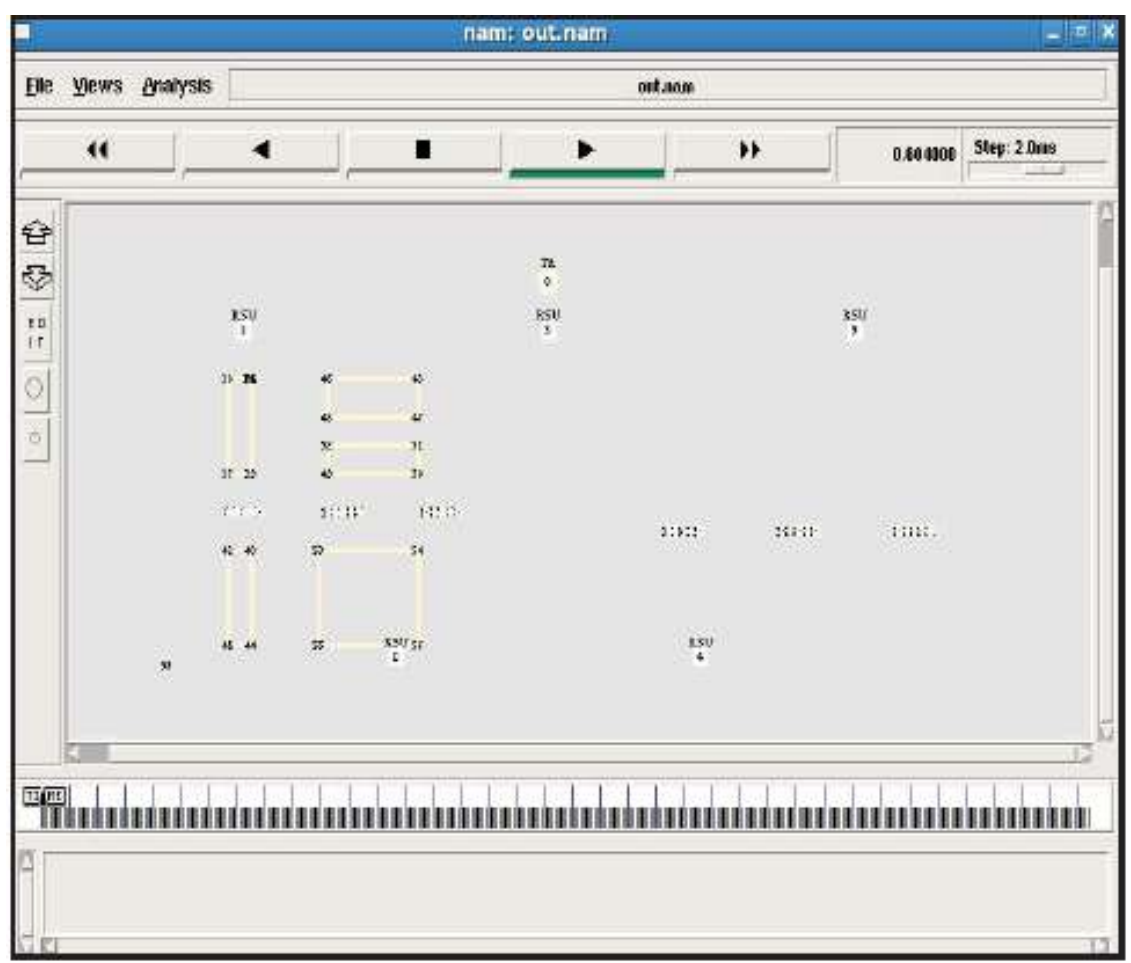

Figure 5. Parking System Scenario in NS-2

For providing efficient and friendly parking scheme (or space), our implemented parking scenario (simulation model) in NS-2 simulator can be showed as in Figure 5.

\subsection{Metrics used to Evaluate Simulation Results}

In our approach, five values for each parking lot are distributed in the network, namely timestamp, total capacity of the parking lot, number of parking places that are currently occupied, and finally, two rates: the arrival rate of vehicles, and the parking rate. Parking rate is an additional feature of our proposed (ISPAS) approach. The parking rate is the converse of the average time for which a vehicle stays on its parking area before it leaves again. These rates are measured at the parking garages which are fixed by parking owner. Presently let $\mathrm{T}$ be the pool or supply of people per unit area in travel to their goals, $\mathrm{C}$ be the pool of people per unit area cruising for parking, and $\mathrm{P}$ be the load of on-road parking spots per unit range, which breaks even with the pool of people stopped per unit zone when parking is immersed. The rate of entry into the network per unit time-area, or proportionally the excursion request per unit time-area is $\mathrm{D}$. Trip request relies upon the maximum of a trip, which equals the cost of in-transit travel time. In our approach, we measure users IN time and OUT time in a parking lot. For that, let $t$ denote the in-transit travel time per unit distance, which individual treats as exogenous but it is determined by how congested the roads are, as part of the overall equilibrium. Consider each of the pools in turn. The rate of 
change of the size of the in-transit pool equals the entry rate, $D$, minus the exit rate, $E$ (see equation 7). Thus,

$$
T(u)=D(u)-E(u) \ldots \ldots \ldots \ldots
$$

where $u$ denotes time. With saturated parking, the rate of change of the size of the cruising-for parking pool, $C$, equals the entry rate into the cruising-for-parking pool, which equals the exit rate from the in-transit pool, minus the exit rate from the cruising-for-parking pool, Z (refer equation 8).

$$
C(u)=E(u)-Z(u)
$$

This work examines only the steady state. In the steady state, the exit rate from the intransit pool equals the pool size divided by an individual's time in the pool, so that equation (7) becomes

$$
\mathrm{D}=\frac{\mathrm{T}}{\mathrm{mt}} \ldots \cdots
$$

And with saturated parking the exit rate from the cruising-for-parking pool equals the exit rate from parking so that equation (8) becomes

$$
\frac{\mathrm{T}}{\mathrm{mt}}=\frac{\mathrm{P}}{\mathrm{l}} \ldots \ldots
$$

These steady-state relationships with saturated parking are displayed in Figure 6. We refer to equation 9 as the in-transit pool steady-state condition and to equation 10 as the cruising-for parking pool steady-state condition.

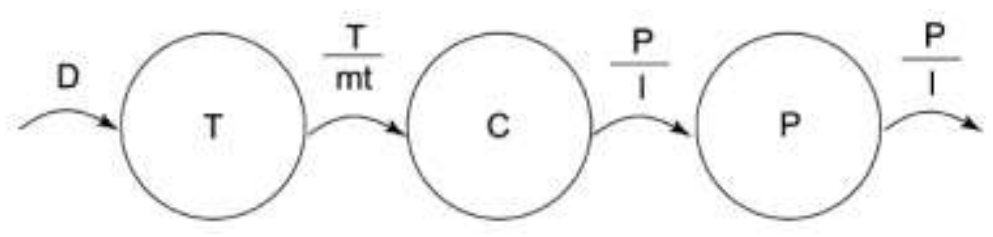

\section{Figure 6. Steady-State Stocks and Flows with Saturated Parking}

Where $T=$ number of cars in transit (per unit area).

$C=$ number of cars cruising for parking (per unit area).

$P=($ On-street $)$ parking spaces $=$ number of cars parked $($ per unit area $)$.

$D=$ Demand inflow (per unit area).

$T / m t=$ Exit rate from in-transit pool $=$ entry rate into cruising-for-parking pool (per unit area).

$P / l=$ Exit rate from cruising-for-parking pool $=$ entry rate into parking pool $=$ exit rate from parking pool $=$ throughput (per unit area).

Now the steady-state full trip price, $F$, is

$$
F=\rho(m t+C l P)+(\rho+f) l
$$

Where $\rho$ is the value of time and $f$ is the on-street parking fee per unit time. The full trip price is the sum of the in-transit travel time cost, the expected cruising-for-parking time cost. Now communication being held among parking authority and vehicle users (i.e., OBU, RSU, and TA) can be notified in several metrics like;

Packet delivery ratio (PDR): Packet delivery ratio is the ratio to evaluate the performance of the simulation. It is the ratio of the packets that successfully reached at destination. 


$$
\text { Packet delivery ratio }=\frac{\text { Total no. of packets delivered }}{\text { Total no. of packets transferred }} * 100 \ldots \ldots \ldots \ldots \ldots
$$

Further, as discussed in section 3, irregularity of vehicles in VANET applications (e.g., parking) can be computed as:

$$
\begin{aligned}
& \text { Total no of available parking space in an } \frac{\text { area }}{\operatorname{street}(\mathrm{N})} \\
& =\mathrm{n} 1+\mathrm{n} 2+\mathrm{n} 3 \ldots \ldots+\mathrm{nk} \ldots \ldots \ldots
\end{aligned}
$$

(Where $\mathrm{n}_{1}, \mathrm{n}_{2} \ldots . \mathrm{n}_{\mathrm{k}}$ are parking spaces).

Now to maintain (or prevent) vehicles from collisions among several users / saving reserved parking slot for a user (see equations 14-15).

$$
\begin{aligned}
& \mathrm{p}(\text { ttot })=\frac{\text { salt }}{(\text { ttot }+ \text { salt })} \\
& Q=\text { asm } * \text { sts }+ \text { wat }
\end{aligned}
$$

In equation 15 , Where $\mathrm{Q}$ is a time penalty taken if space $\mathrm{b}$ (intermediary/ reserved parking place) is not available when the vehicle arrives there. In equation 14, ttot represents the accrued time. The "space average life-time" (salt) is the average period, i.e., a parking space remains free once vacated. The average number of parking spaces (visited by a user before occupying a spot) available is designated as "average spot missed" (asm). Based on the data (updated parking slots), we compute the following two factors: first, the average time to drive from all spaces to all others or "spot to spot" (sts) and second, the "average walk time" (wat) from all spaces to d (refer equations 14 and 15). To provide implement results in efficient parking schemes, four other parameters like False positive (FP), True positive (TP), False negative (FN) and True negative (TN) also used (refer equations 1619). True positive (TP) is, if the analyzed event is correctly classified as intrusion/ malicious.

$$
\begin{aligned}
& \text { True Positive Rate (or sensitivity)(TPR) }=\frac{\mathrm{TP}}{\mathrm{TP}+\mathrm{FN}} \\
& \text { False Positive Rate }(\mathrm{FPR})=\frac{\mathrm{FP}}{\mathrm{FP}+\mathrm{TN}} \ldots \ldots \ldots \ldots \ldots \ldots \ldots \ldots \ldots \ldots \ldots \ldots \ldots \ldots \ldots \\
& \text { True negative rate (or specificity) }(\mathrm{TNR})=\frac{\mathrm{TN}}{\mathrm{FP}+\mathrm{TN}} \\
& \text { False Negative Rate (FNR) }=\frac{\mathrm{FN}}{\mathrm{TP}+\mathrm{FN}} \ldots \ldots \ldots \ldots \ldots \ldots
\end{aligned}
$$

Here False positive (FP) is, if the analyzed event is innocuous (or "clean') from the perspective of security, but it is classified as malicious. True negative (TN) is, if the analyzed event is correctly classified as normal/ innocuous. False negative (FN) is, if the analyzed event is malicious but it is classified as normal/ innocuous. In general, Four situations exist in this context, corresponding to the relation between the result of the detection for an analyzed place ("normal" vs. "intrusion") and its actual nature ("innocuous" vs. "malicious"). These situations are: trust positive, false negative etc. can be discussed in simple words like; Positive $=$ identified and negative $=$ rejected. Therefore: True positive means correctly identified, False positive means incorrectly identified, True negative means correctly rejected and False negative refers to incorrectly rejected. We use privacy metrics in our approach (proposed work): a) time needed to reach a free parking 
space, $b$ ) time required to walk from the selected space to the final destination and c) the percentage of vehicles that managed to park.

\subsection{Simulation Results}

Figure 7 depicts the effect of $Q T$ Clustering with 3 different values for quality threshold QT hres. This threshold expresses the maximum radius a cluster may display. We choose ave and std as the parameters which affect the maximum radius. The three levels of clustering are designated as:

- Cluster1: QT hres = ave/ 3

- Cluster2: QT hres = ave/ $3+$ std $/ 2$

- Cluster3: QT hres $=$ ave/ $3+2 *$ std

In Figure 7, $\mathrm{x}$-axis expresses the quantity of free parking lots/ spots known by the vehicle that determine the trip around them. The y-axis communicates the execution time for the calculation of the trip in milliseconds and logarithmic scale. The execution time required by Algo. 2 and 3 which finds the ideal excursion (to goal), builds exponentially to the quantity of known stopping places. The execution time for Cluster1 likewise rises when the quantity of parking spots increases, as the quantity of groups that are formed becomes larger. Cluster 2 and Cluster 3 frame less bunches than Cluster1 and has decreased execution times.

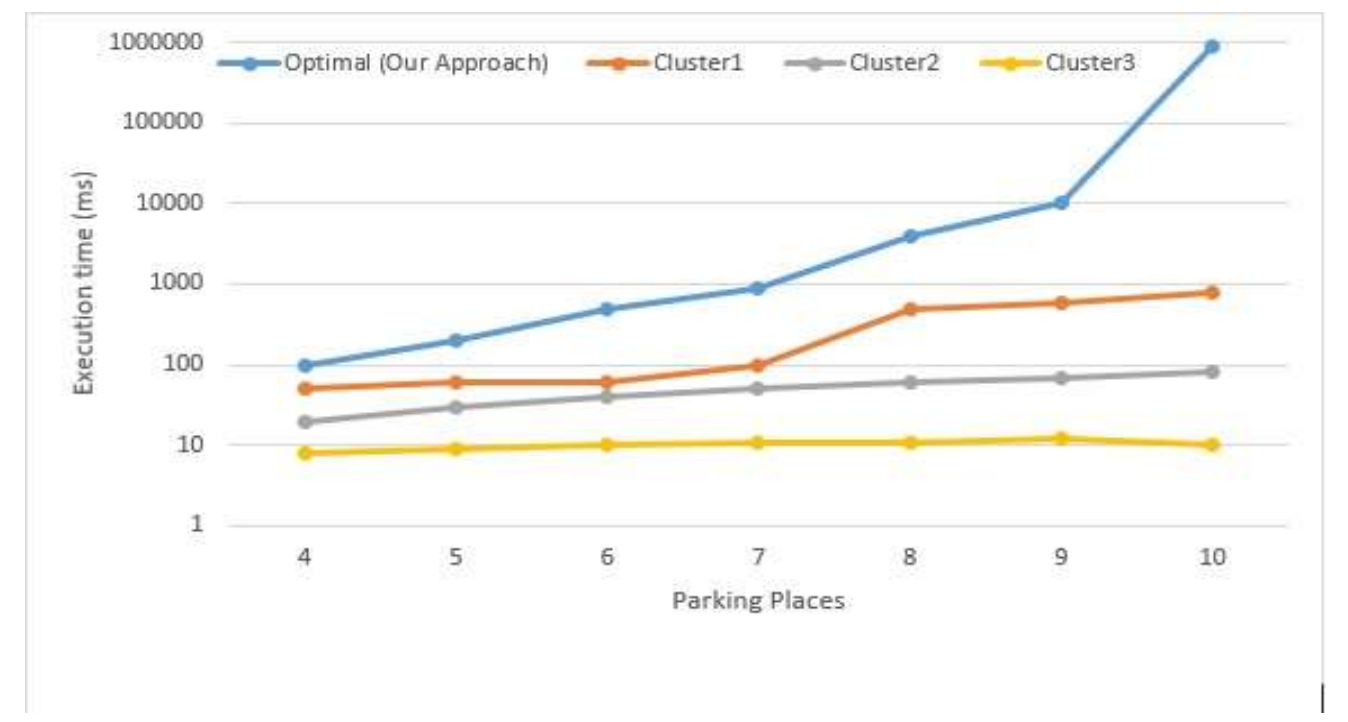

\section{Figure 7. Time to Compute the Trip around Parking Places for the Optimal and Three Clustering Levels}

Figure 8 shows that, cutting off gives an essentially preferable result over the grouping strategy (or clustering method), for all the clustering levels, while the execution time is marginally slightly more in most cases. This result shows that it is ideal to locate the ideal (optimal) trip for a number $\mathrm{L}$ of representatives for the entire parking spot set, and after that embed whatever is left in spaces. It noticed (and update) in our system and then for another users, we apply but first approach to provide allocation of vacant parking spaces to the respective vehicle users. 


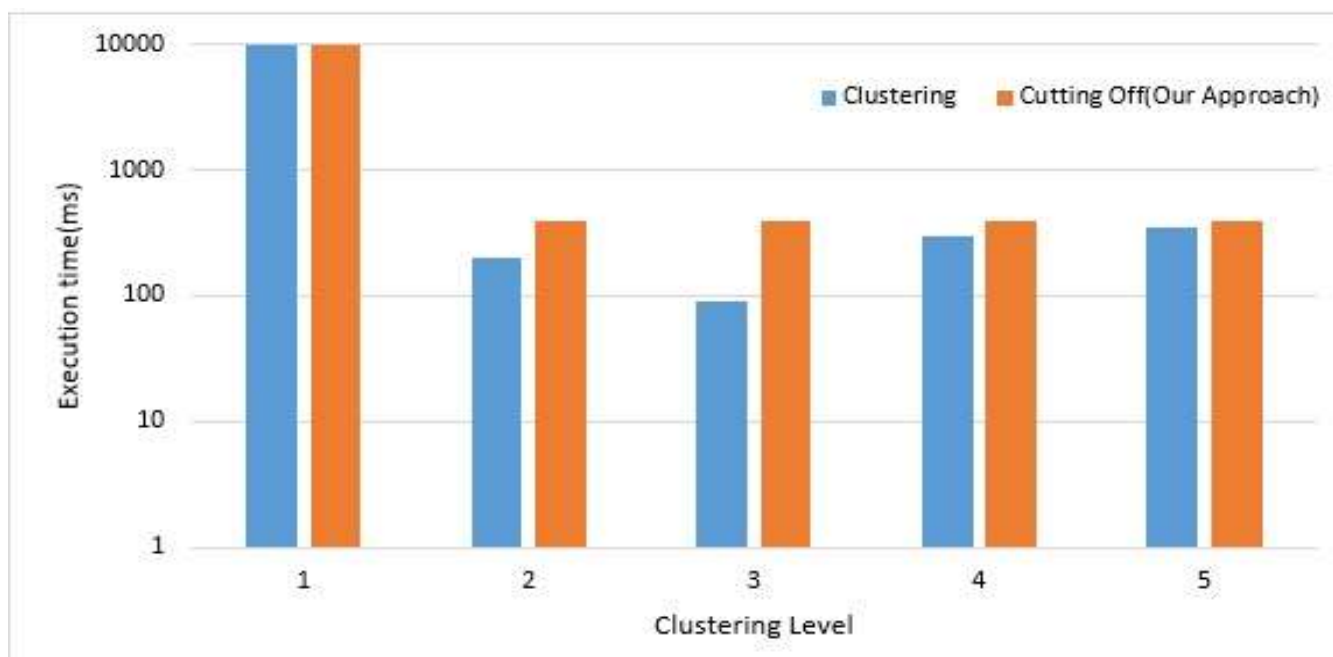

Figure 8. Time to Compute the Trip around Parking Places for Clustering and Cutting-Off Methods

Figure 9 delineates the computational overhead and the deviation from the ideal trip when Live-Mode is being used. In Figure 9, a vehicle re-adjusts trajectory, for a number of updates. The execution time for taking care of these updates, begins from $70 \mathrm{~ms}$ for one or two updates, then increases directly, and finally achieves $700 \mathrm{~ms}$ for 10 updates.

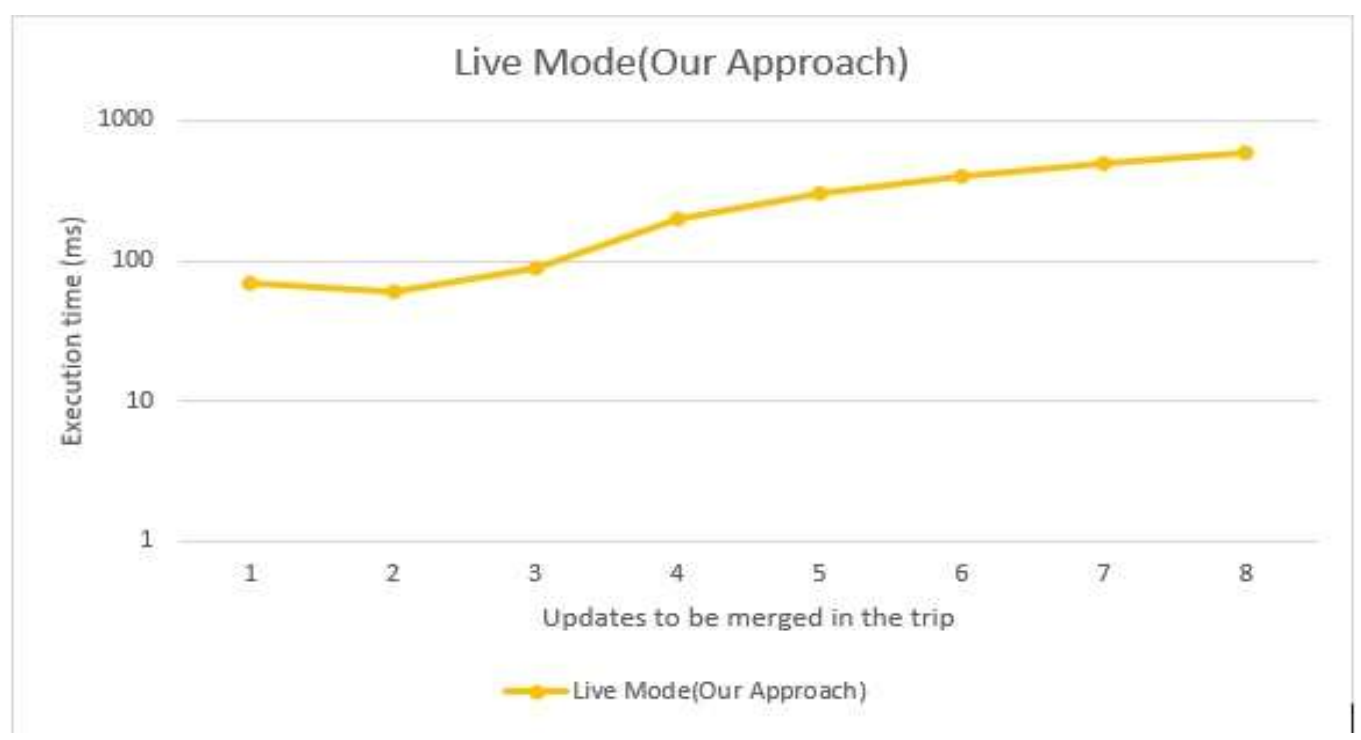

Figure 9. Execution Time for Merging a Number of Updates, Using the Live Mode

Figure 9 shows that Live-Mode includes low computational overheads and the rebalanced (re-adjusted) it for an ideal trip. When grouping is performed with a large number or when cutting off is performed with a little number of representative, the formed route might be impressively more terrible than the ideal one. The most effective approach to keep the extent of the issue little and achieve a close ideal solution is: first convey grouping with a little range and utilize $\mathrm{k}$-medoids to choose $\mathrm{k}$ representative clusters (groups). 


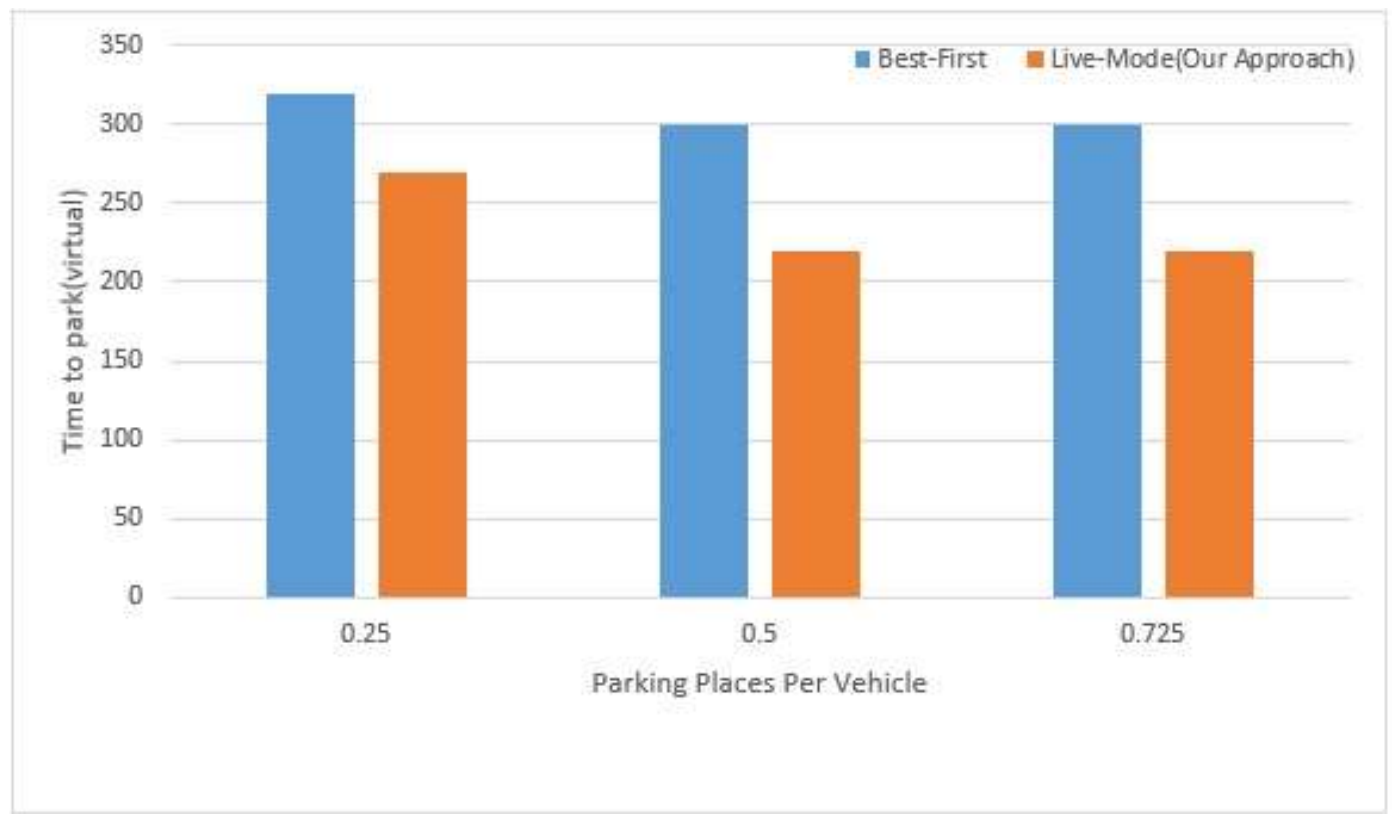

Figure 10. Time to Find a Parking Space Free for the Live-Mode and BestFirst Approaches

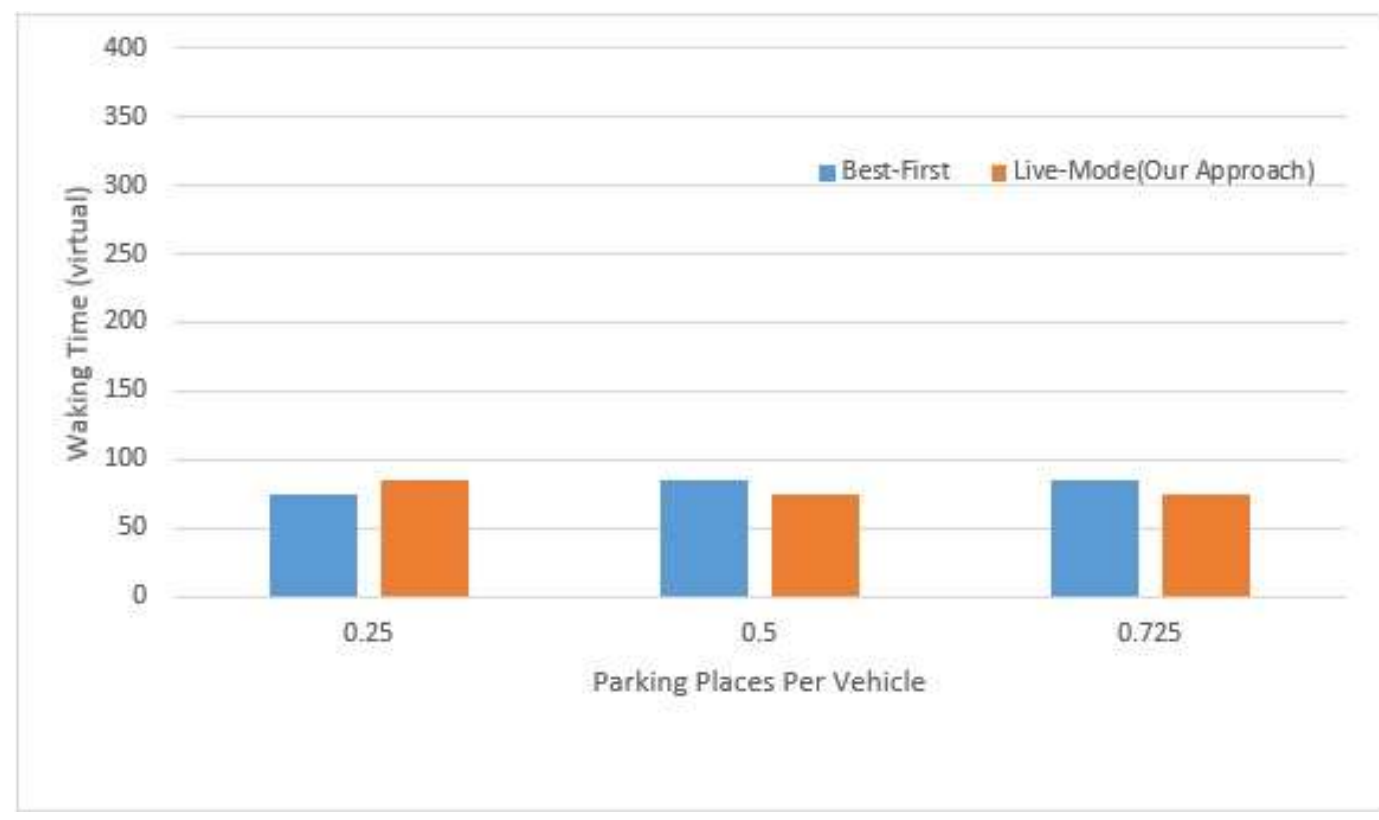

Figure 11. Walking Time to the Parking Place for Live-Mode and Best-First Approaches

Figures. 10, 11 and 12 respectively demonstrate the average time expected to locate a free parking spot, the time expected to walk from that parking spot to the final destination, and the rate of vehicles that found a free space utilizing the two methodologies. Live-Mode shows better results in every one of the three cases. 


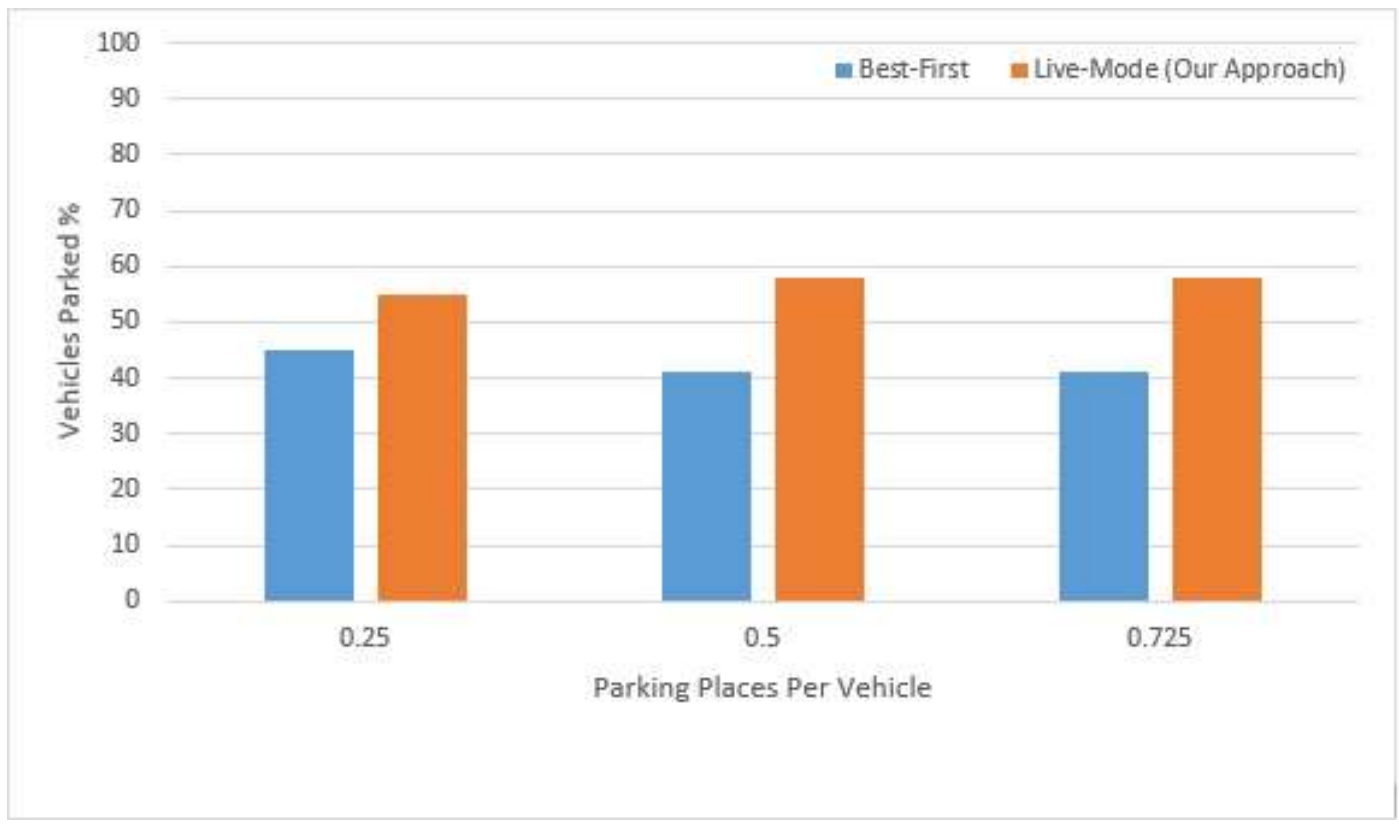

Figure 12. Percentage of vehicles that Found a Parking Space in Live-Mode
and Best-First Approaches

Vehicles that work with Live-Mode, decide to start with visiting all territories, where high densities of parking spots is available. Conversely, Best-First vehicles choose to visit spots that are their best choice with respect to user's final destination and current position, regardless of the possibility that these spots lie on regions (areas) with low-density of accessible spaces. The fierce competition among vehicles is utilizing the Best-First approach. It does not permit any critical change when the proportion of spaces per vehicle increments. Figure 10 have been analysed for all space proportions, i.e., Live-Mode accomplishes better normal time (if contrasted) in compare to Best-First approach. The greatest increases show up for proportions 0.5 and 0.725 where Live-Mode can help vehicle adequately go around empty spots. For space proportion 0.25 , the distinction of time required to park between Live-Mode and Best-First is perceptibly smaller because of higher rivalry for spaces. Figure 11 delineates the walking time to the destination is close for both methodologies (in all cases). We would like to expect that a vehicle user is utilizing the Best-First approach. And it would perform better to those who are empowered with LiveMode and tries to forcefully choose a spot near to a destination. Hence our approach (live, best and clustering) work efficiently in different-different scenario (e.g., for high density, low density etc.) and with different-different users requirements (for e.g., space near to his destination, security protected etc.). Note that, here we do not preserve privacy of a user using an algorithm or mechanism, but we tried to avoid using sensor to preserve or protecting of privacy of users in a parking area. Figure 12 shows, the percentage rate of vehicles finding a free space reliably stays higher for the Live-Mode approach. The results of Figure 12 are in accordance with those exhibited in Figure 10 and 11 simplify the differences for ratios 0.5 and 0.725 .

Hence this section discusses about experimental setup, performance metrics to compute or provide an efficient solution for parking problem to vehicle users. We hope that this scheme will be really helpful for real world users. Now next section concludes this work in brief and draws a road ahead for future.

\section{Conclusions and Future Work}

Due to the industrialization of the world, several problems have been raised in urban areas like; increasing accidents and congestion, pollution, slow paced city development and 
mismanagement of the available parking space etc. some problem like: reduction of total number of vehicle (or pollution) over road network can be controlled (solved) with providing vacant parking slots to vehicle users over the road/ street network. When several vehicles (public transport) move over the road, then after completing their journey, everyone need safe and secure space to park their vehicle. As discussed in related work, several mechanisms for providing efficient parking have been proposed in the past decade. But none of the approach is sufficient in vehicular network (due to its dynamic nature) concern user's privacy or does not work efficiently for several problems (like: in response, at the time of system updating etc.). So this work proposed an Intelligent, Smart Parking Allotment System (ISPAS) (using the vehicular ad hoc network's various features). In this work, a new VANET-based friendly and efficient parking scheme for large parking lots is designed, developed and tested using network simulator NS-2. In our scheme, RSUs installed across a parking lot which can survey (analyze) the whole parking lot, and provide two convenient services to drivers' (vehicles), i.e., real-time parking navigation and friendly parking information dissemination. Extensive simulations have also been conducted to demonstrate that the proposed scheme can efficiently reduce the searching time delay for an available vacant parking space, and subsequently save the fuels and driver's parking time. In this paper, simulation of VANET used for a friendly parking system and implemented with user's requirements and its tested founded satisfactory.

We know due to dynamic nature of vehicle (or VANET), several problems raised here. So as future work, we need to protect user's information over parking duration/ protect privacy of users and maintain trust among certified authorities and vehicle users. Also providing efficient parking to different sizes of vehicles (e.g., cars, bus, etc.) and different users (users have different preferences) can be considered as a future work. Hence the future work includes more extensive simulations for this proposed system.

\section{Conflict of Interests}

The authors declare that there is no conflict of interests regarding the publication of this paper.

\section{Competing Interests}

Authors have declared that no competing interests exist.

\section{References}

[1] A. K. Tyagi and N. Sreenath, "Providing Trust Enabled Services in Vehicular Cloud Computing (extended version)", in proceeding of ACM/ International Conference on Informatics and Analytics (ICIA), 25-26 August, Pondicherry, India, (2016).

[2] A. K. Tyagi and N. Sreenath, "Vehicular Ad Hoc Networks: New Challenges in Carpooling and Parking Services", in proceeding of International Conference on Computational Intelligence and Communication (CIC), Pondicherry, India 2016, vol. 14, (2016),

[3] R. Panayappan and J. M. Trivedi, "VANET-based approach for parking space availability", in Proceedings of the Fourth ACM International Workshop on Vehicular Ad Hoc Networks, Montreal, Quebec, Canada, (2007), pp. 75-76.

[4] G. Yan, W. Yang, D. B. Rawat and S. Olariu, "SmartParking: A Secure and Intelligent Parking System," in Intelligent Transportation Systems Magazine, IEEE, vol. 3, (2011), pp. 18-30.

[5] V. Verroios, V. Efstathiou and A. Delis, "Reaching available public parking spaces in urban environments using ad hoc networking”, in Mobile Data Management (MDM), 12th IEEE International Conference on., (2011), pp. 141-151.

[6] J. B. Kenney, "Dedicated Short-Range Communications (DSRC) Standards in the United States", in Proceedings of the IEEE, vol. 99, (2011), pp. 1162-1182.

[7] A. Ghazy and T. Ozkul, "Design and simulation of an artificially intelligent VANET for solving traffic congestion", in Mechatronics and its Applications, ISMA '09. 6th International Symposium on, (2009), pp. $1-6$.

[8] J. Sun, Y. Zhang and J. Fan, "Towards a context-aware middleware in smart car space", in Genetic and Evolutionary Computing (ICGEC), Fourth International Conference on, (2010), pp. 276-279. 
[9] Alhammad, Abdulmalik, Siewe and Francois, "An InfoStation-Based Context-Aware On-Street Parking System", IEEE, (2013).

[10] M. Hosseinnezhad Ghavifekr and A. Chodari Khosrowshahi, "Parking based Data Replication in VANET", Indian Journal of Science and Technology, vol. 8, no .27, (2015).

[11] M. Caliskan, D. Graupner and M. Mauve, "Decentralized Discovery of Free Parking Places", in Proc. of the 3rd Int. Workshop on Vehicular Ad hoc Networks, ser. VANET '06. Los Angeles, CA: ACM, (2006), pp. 30-39.

[12] T. Delot, N. Cenerario, S. Ilarri and S. Lecomte, "A Cooperative Reservation Protocol for Parking Spaces in Vehicular Ad-hoc Networks", in Proc. of the 6th Int. Conf. on Mobile Technology, Application and Systems (Mobility'09). Nice, France: ACM, (2009), pp. 1-8.

[13] M. Caliskan, A. Barthels, B. Scheuermann and M. Mauve, "Predicting Parking Lot Occupancy in Vehicular Ad hoc Networks", in Proc. of the IEEE Int. Conf. on Vehicular Technology Conference (VTC'07). Dublin, Ireland: IEEE, (2007), pp. 277-281.

[14] A. Khekdar and V. Kumar, "Self-Synchronizing Moving Objects Using Contextual Information", in Proc. of the 2010 Int. Conf. on Information and Knowledge Engineering (IKE'10), Las Vegas Nevada, USA: CSREA Press, (2010), pp. 330-335.

[15] V. Verroios, K. Kollias, P. K. Chrysanthis and A.Delis, “Adaptive Navigation of Vehicles in Congested Road Networks", in Int. Conf. on Pervasive Services (ICPS'08), Sorrento, Italy: ACM, (2008).

[16] E. Kokolaki, M. Karaliopoulos and I. Stavrakakis, "Value of information exposed: wireless networking solutions to the parking search problem", in Proc. of the 8th Int. Conf. on Wireless On-demand Network Systems and Services (IFIP/ IEEE WONS), Bardonecchia, Italy, (2011).

[17] S. Mathur, T. Jin and N. Kasturirangan, "ParkNet: drive-by sensing of road-side parking statistics", in Proc. of the 8th Int. Conf. on Mobile Systems, Applications, and Services (MobiSys '10). San Francisco, USA: ACM, (2010), pp. 123-136.

[18] J. Boehle, L. Rothkrantz and M. V. Wezel, "CBPRS: A City Based Parking and Routing System”, ERIM Report Series Reference No. ERS- 2008-029-LIS, (2008).

[19] R. Lu and X. Lin, "SPARK: A New VANET-based Smart Parking Scheme for Large Parking Lots”, IEEE INFOCOM 2009 proceedings.

[20] H. Zhao, L. Lu and C. Song, "IPARK: Location-Aware-Based Intelligent Parking Guidance over Infrastructureless VANETs", Hindawi Publishing Corporation, International Journal of Distributed Sensor Networks, vol. 2012.

[21] A. K. Tyagi and N. Sreenath, "Never Trust Anyone: Trust-Privacy Trade-Offs in Vehicular Ad hoc Network", British Journal of Mathematics and Computer Science (BJMCS), vol. 19, no. 6, (2016), pp.123.

[22] J. Cui, J. Zhang, H. Zhong and Y. Xu, "SPACF: A Secure Privacy-Preserving Authentication Scheme for VANET with Cuckoo Filter", IEEE Transactions on vehicular technology, vol. 66, no. 11, (2017).

[23] H. Wang, Y. Li, Y. Guo, Y.Agarwal and J.I. Hong, "Understanding the Purpose of Permission Use in Mobile Apps, ACM Transactions on Information Systems (TOIS) - Special issue: Search, Mining and their Applications on Mobile Devices", vol. 35, issue 4, (2017).

[24] S. Chitkara and N. Gothoskar, "Does this App Really Need My Location?: Context-Aware Privacy Management for Smartphones", Proceedings of the ACM on Interactive, Mobile, Wearable and Ubiquitous Technologies, vol. 1, issue 3, (2017). 
International Journal of Security and Its Applications

Vol. 11, No. 12 (2017) 